SU-ITP-98/02

KUL-TF-98/08

hep-th/9801206

\title{
CONFORMAL THEORY OF M2, D3, M5 AND 'D1+D5' BRANES
}

\author{
Piet Claus ${ }^{a}$, Renata Kallosh ${ }^{b}$, Jason $\mathrm{Kumar}^{b}$, \\ Paul K. Townsend ${ }^{c, \star}$ and Antoine Van Proeyen ${ }^{a, \dagger}$ \\ ${ }^{a}$ Instituut voor theoretische fysica, \\ Katholieke Universiteit Leuven, B-3001 Leuven, Belgium \\ ${ }^{b}$ Physics Department, \\ Stanford University, Stanford, CA 94305-4060, USA \\ ${ }^{c}$ Institute for Theoretical Physics, University of California, \\ Santa Barbara, CA 93106, USA
}

\begin{abstract}
The bosonic actions for M2, D3 and M5 branes in their own $d$ dimensional near-horizon background are given in a manifestly $S O(p+1,2) \times S O(d-p-1)$ invariant form $(p=2,3,5)$. These symmetries result from a breakdown of $\operatorname{ISO}(d, 2)$ (with $d=10$ for D3 and $d=11$ for M2 and M5) symmetry by the Wess-Zumino term and constraints. The new brane actions, reduce after gaugefixing and solving constraints to $(p+1)$ dimensional interacting field theories with a non-linearly realized $S O(p+1,2)$ conformal invariance. We also present an interacting two-dimensional conformal field theory on a D-string in the near-horizon geometry of a D1+D5 configuration.

$\dagger$ Onderzoeksdirecteur FWO, Belgium

* On leave from DAMTP, Univ. of Cambridge, UK
\end{abstract}




\section{Introduction}

It is well-known that the anti-de Sitter group is isomorphic to the conformal group in one lower dimension. For this reason the isometry group of anti-de Sitter space acts as the conformal group on its boundary, which is a conformally compactified Minkowski spacetime of one lower dimension. The degrees of freedom at the boundary are the singleton representations of the adS group (see e.g. [1]), so singleton field theories are natural candidates for conformal field theories. If one views the singleton fields as describing fluctuations of the boundary of the $a d S$ space then it is natural to interpret the boundary as a physical object, i.e. a brane: this was the idea behind the 'membrane at the end of the universe' [2]. The connection between singletons and branes can also be understood from the fact that the supergravity solutions representing M2, D3 and M5 branes interpolate between the Minkowski vacuum and a compactification to adS space [3]. On the one hand the field modes trapped near the core are naturally identified as the worldvolume fields of the brane. On the other hand, the core region is also the boundary region of the $a d S$ vacuum. In the past, these ideas motivated various constructions of free (super)conformal field theories on the boundary of $a d S_{p+2}$ or, more generally, conformally flat spacetimes of the same $S^{p} \times S^{1}$ topology [4, 5]. Some recent developments [6, 7, 8, 9] have now brought this program back to life.

One key observation is that if a $p$-brane worldvolume is embedded appropriately in the $a d S_{p+2}$ background then it is not necessary to push the brane to the $a d S$ boundary, as was found to be necessary (for $p=2$ ) in [2]. One way to see this is to observe that for a fixed distance from an infinite static M2 brane (for example) the near-horizon $a d S_{4} \times S^{7}$ geometry [3] becomes a better and better approximation as the membrane number $N$ (i.e. its charge, classically) increases [7]. Now, given say 1001 parallel coincident M2 branes we can move one of them away; if it is static and remains parallel to the other 1000 membranes it will feel no force, but it will now appear to be a 'test' brane in the $a d S_{4} \times S^{7}$ background of the other 1000. Since the distance of this test brane from the 'source' branes is arbitrary there is an apparent contradiction with the result of [2], which we resolve in Appendix A. Here we need note only that the implication of the above argument, i.e. that in an $a d S_{4} \times S^{7}$ background there exists a solution of the M2 brane equations that describes a static infinite flat membrane at arbitrary ad $S$ radius, is correct.

The corresponding gauge-fixed M2 brane action must be a 3-dimensional field 
theory with a Minkowski vacuum. The symmetries of this theory are those of the $O S p(8 \mid 4)$ isometry supergroup of the background, which we may now interpret as a superconformal group acting on worldvolume fields. We thus find a 3-dimensional superconformal field theory on the worldvolume of the M2 brane, which turns out to be an interacting field theory. In the limit that the M2 brane is pushed to the adS boundary we recover the free superconformal field theory on the 'membrane at the end of the universe'. The observation that the membrane need not be placed at the boundary is therefore crucial in allowing the introduction of interactions into (super)singleton field theories.

The ideas just sketched have been used recently to make some progress towards the construction of interacting superconformal field theories on brane worldvolumes [7, 8, 9], in particular on the worldvolumes of the M2, D3 and M5 branes. The essential features shared by these cases is that the corresponding static supergravity p-brane solution has a non-singular degenerate Killing horizon with respect to a Killing vector field that is timelike near spatial infinity [10, with the near horizon geometry being $a d S_{p+2} \times S^{d-p-2}$ [3]. In addition there is a non-vanishing $(p+1)$-form gauge potential. The (super)conformal invariance of the action for a test brane in this background was conjectured in [0, 8], where it was pointed out that the adS radius of curvature $R$ is also the coupling constant of the worldvolume field theory. More precisely, the coupling constant is the dimensionless ratio $R /\langle r\rangle$, where $\langle r\rangle$ is the distance of the brane from the horizon, so that the weak coupling limit $R \rightarrow 0$ (at fixed $\langle r\rangle$ ) is equivalent to the 'end of the universe' limit $\langle r\rangle \rightarrow \infty$ (at fixed $R$ ). The conformal invariance of the action after a truncation to radial adS excitations was also verified in [7, 8]. Here we shall fully establish the conformal invariance of the bosonic actions by systematically exploiting the fact that this symmetry follows directly from the isometries of the background. We thereby find new (classically) conformal invariant field theories on the worldvolumes of the M2, D3 and M5 branes. Our method is also novel. It involves a reformulation of the (worldvolume diffeomorphism invariant) brane action in which the $S O(d, 2)$ conformal invariance is manifest, being realized linearly as transformations in a space of $d+2$ dimensions. There is therefore a possible connection to a $(10+2)$ dimensional F-theory [1] underlying the IIB branes and an 11+2 dimensional S-theory 12 underlying the M-branes (see [13] for a recent review of supersymmetry in dimensions beyond eleven). In our case, however, the additional dimensions serve to linearly realize conformal invariance rather than a higher-dimensional Lorentz 
invariance . The manifest conformal invariance is broken when one attempts, by gauge-fixing and solving constraints, to express these actions in terms of only the 'physical' fields, but the conformal invariance is still there; it is just non-linearly realized. The choice of background is clearly crucial to this result because symmetries of brane actions correspond to isometries of the background. For a flat background, for example, it has been established [6] that the worldvolume field theory is (super)conformal only in the linearized approximation. We understand now the conformal symmetries of this linearized approximation as the limit $R \rightarrow 0$ of the conformal action in adS background.

We use essentially the same methods to find an interacting conformal invariant (classical) field theory on the worldvolume of a D-string in the near-horizon background of a D-string parallel to a D5-brane, we call this the 'D1+D5' case. The near-horizon geometry of various intersecting brane configurations has been discussed previously in [15, 16, 17, 18. In our case it is $a d S_{3} \times S^{3} \times E^{4}$, which makes it essentially equivalent to the near-horizon $a d S_{3} \times S^{3}$ geometry of the $d=6$ self-dual string $10 \|$. Thus, the D1+D5 case is similar to the $d=6, p=1$, realization of the $a d S_{p+2} \times S^{d-p-2}$ class of geometries near brane horizons. In addition, as we show here, there is a partial restoration of supersymmetry near the horizon (corresponding to total restoration in the context of the $d=6$ self-dual string). This complements the already known restoration of supersymmetry near the M2, D3 and M5 brane horizons. Thus, in each of the four cases (M2, D3, M5 and D1+D5) the $S O(d, 2) \times S O(d-p-1)$ isometry of the background is actually the bosonic subgroup of a supergroup. The latter are

$$
\begin{aligned}
M 2: & O S p(8 \mid 4) \\
D 3: & S U(2,2 \mid 4) \\
M 5: & O S p(6,2 \mid 4) \\
D 1+D 5: & S U(1,1 \mid 2) \times S U(1,1 \mid 2) .
\end{aligned}
$$

These supergroups have been indicated by Nahm [19 as super-adS and superconformal symmetries. The last one is listed among the $a d S_{3}$ supergroups of [20], where the supersingleton representations can also be found. We therefore expect that all the results presented here have supersymmetric ex-

\footnotetext{
${ }^{1}$ It follows that our actions should not be viewed as (bosonic) realizations of the super p-branes in spacetimes of non-Lorentzian signature proposed in [14].
} 
tensions in which these isometry supergroups are realized as superconformal symmetries on the brane, but we leave this to a future investigation.

In section 2 we will consider a class of world-volume actions in $a d S_{p+2} \times$ $S^{d-p-1}$ backgrounds. In this part there are only scalar fields. We present an $I S O(d, 2)$ symmetric form of the action with Lagrange multiplier terms which break the symmetry down to $S O(p+1,2) \times S O(d-p-1)$. We show how this group is realized as a rigid extended superconformal symmetry of the gaugefixed action. In section 3 this general result is applied, with the necessary modifications, to the $M 2, D 3, M 5$ and $D 1+D 5$ branes. The Wess-Zumino terms are given in each case and the expansion of the full action about the quadratic term is given for the first few terms (this is the action in the 'small velocities' approximation). Section 4 has a short discussion of the results and possible developments.

In Appendix A we explain an apparent contradiction with [2], where it was concluded that supersymmetric and static membranes only exist when the brane is located at static infinity. We show that their actions are included in our general action, and the difference of solutions is due to different interpretations to the word 'static', related to the existence of more than one timelike Killing vector field. In Appendix B we verify the enhancement of supersymmetry near the horizon of the D1+D5 configuration. In Appendix 9 we explain how the Born-Infeld actions are expanded in the approximation of small velocities.

\section{Conformal invariance 'on the brane'}

\subsection{World-volume action}

Let $\sigma^{\mu},(\mu=0,1, \ldots, p)$ be coordinates on the $(p+1)$-dimensional world volume $W$ of a $p$-brane, with metric $g_{\mu \nu}$ induced from a metric $\mathcal{G}$ on a $d$ dimensional spacetime $\mathcal{M}_{d}$. We want to consider $p$-brane actions of the form

$$
S=-\int_{W} d^{p+1} \sigma \sqrt{-\operatorname{det} g_{\mu \nu}}+\int_{W} A,
$$

where $A$ is a $(p+1)$-form induced from a $(p+1)$-form gauge potential $\mathcal{A}$ on $\mathcal{M}_{d}$. The first term is invariant under the isometries of the background spacetime metric $g$, which are generated by killing vector fields $\xi$. The second term will also be invariant provided that, for all $\xi$,

$$
\mathcal{L}_{\xi}(d \mathcal{A})=0
$$


where $\mathcal{L}_{\xi}$ is the Lie derivative with respect to $\xi$. We choose the metric on $\mathcal{M}_{d}$ to be the $S O(p+1,2) \times S O(d-p-1)$ invariant metric on $a d S_{p+2} \times S^{d-p-2}$. In horospherical coordinates for $a d S$, and hyperspherical coordinates for $S^{d-p-2}$, this metric is

$$
d s^{2}=\phi^{2} d x^{m} \eta_{m n} d x^{n}+(w R)^{2}\left(\frac{d \phi}{\phi}\right)^{2}+R^{2} d^{2} \Omega,
$$

where $d \Omega^{2}$ is the $S O(d-p-1)$-invariant metric on the unit $(d-p-2)$-sphere. The constant $R$ is clearly the radius of curvature of the $S^{d-p-2}$ factor. It is also proportional to the $a d S$ radius of curvature $w R$ and the constant $w$ is chosen to be

$$
w=(p+1) /(d-p-3) .
$$

We note, in passing, that this metric is conformally flat (vanishing Weyl tensor) in those cases for which $w=1$. For future use we note that in terms of a radial coordinate $r$ defined by

$$
\phi=\left(\frac{r}{R}\right)^{\frac{1}{w}}
$$

the metric becomes

$$
d s^{2}=d X^{M} \mathcal{G}_{M N} d X^{N}=\left(\frac{r}{R}\right)^{\frac{2}{w}} d x^{m} \eta_{m n} d x^{n}+\left(\frac{R}{r}\right)^{2}\left[d r^{2}+r^{2} d \Omega^{2}\right] .
$$

In these coordinates the infinitesimal action of the $S O(p+1,2)$ isometry group is

$$
\begin{aligned}
\delta_{a d S}(\xi) x^{m} & =-\hat{\xi}^{m}(x, r)=-\xi^{m}(x)-(w R)^{2}\left(\frac{R}{r}\right)^{\frac{2}{w}} \Lambda_{K}^{m}, \\
\delta_{a d S}(\xi) r & =w \Lambda_{D}(x) r,
\end{aligned}
$$

where

$$
\begin{aligned}
\xi^{m}(x) & =a^{m}+\lambda_{M}^{m n} x_{n}+\lambda_{D} x^{m}+\left(x^{2} \Lambda_{K}^{m}-2 x^{m} x \cdot \Lambda_{K}\right) \\
\Lambda_{D}(x) & =\frac{1}{d} \partial_{m} \xi^{m}=\lambda_{D}-2 x \cdot \Lambda_{K}
\end{aligned}
$$

and $a^{m}, \lambda_{M}^{m n}, \lambda_{D}, \Lambda_{K}^{m}$ are the constant parameters associated with translations $P_{m}$, Lorentz transformations $M_{m n}$, dilatations $D$ and special conformal 
transformations $K_{m}$. The $a d S_{p+2}$ isometries are given in terms of the parameters of the conformal transformations in $p+1$ dimensions. More about the expressions for $\xi^{m}$ and $\Lambda_{D}$ can be found in section 2 of [6]. These transformations may be found directly by solving the Killing condition to find the Killing vector fields $\xi$, and then computing their action on the coordinates.

Alternatively, we may proceed as follows. We introduce a space $\mathcal{S}_{d+2}$ of dimension $(d+2)$ with coordinates

$$
\begin{aligned}
X^{\hat{M}}= & \left\{X^{\hat{m}}=\left\{X^{m}, X^{-}, X^{+}\right\}, X^{\hat{m}^{\prime}}\right\} \\
& \left(m=0,1, \ldots, p ; \quad \hat{m}^{\prime}=p+1, \ldots, d-1\right)
\end{aligned}
$$

and a flat metric with (mostly plus) signature $(d, 2)$, i.e.

$$
d s^{2}=d X^{m} \eta_{m n} d X^{n}-d X^{+} d X^{-}+d X^{\hat{m}^{\prime}} \delta_{\hat{m}^{\prime} \hat{n}^{\prime}} d X^{\hat{n}^{\prime}}
$$

where $\eta$ is the $(p+1)$ Minkowski metric and $\delta$ is the $(d-p-1)$ Euclidean metric. The $a d S_{p+2} \times S^{d-p-2}$ submanifold is the intersection of the two hypersurfaces defined by the constraints

$$
\begin{aligned}
& T_{1} \equiv X^{m} \eta_{m n} X^{n}-X^{+} X^{-}+(w R)^{2}=0, \\
& T_{2} \equiv \delta_{\hat{m}^{\prime} \hat{n}^{\prime}} X^{\hat{m}^{\prime}} X^{\hat{n}^{\prime}}-R^{2}=0 .
\end{aligned}
$$

The $I S O(d, 2)$ invariance of the metric on $\mathcal{S}_{d+2}$ is broken by these constraints to $S O(p+1,2) \times S O(d-p-1)$. The first constraint may be solved by setting

$$
\begin{aligned}
X^{-} & =\phi, \\
X^{m} & =\phi x^{m}, \\
X^{+} & =\frac{(w R)^{2}+\phi^{2} x^{2}}{\phi} .
\end{aligned}
$$

The second constraint defines a $(d-p-2)$-dimensional hypersphere of radius $R$ in $\mathbb{E}^{d-p-1}$ and can be solved in terms of hyperspherical coordinates? $\theta_{i}$

$$
X^{\hat{m}^{\prime}}=\left\{R \cos \theta_{1}, R \cos \theta_{2} \sin \theta_{1}, \ldots, R \prod_{i=1}^{d-p-2} \sin \theta_{i}\right\} .
$$

\footnotetext{
${ }^{2}$ Note that the $\theta$ 's do not cover the whole sphere and we need at least two coordinate patches. However, we will only work in one patch.
} 
The induced $d$-metric on the submanifold defined by these constraints is precisely the metric (2.3). The $S O(p+1,2)$ invariance is linearly realized on the $X^{\hat{m}}$ coordinates,

$$
\delta X^{\hat{m}}=-\Lambda_{\hat{n}}^{\hat{m}} X^{\hat{n}} ; \quad \Lambda_{\hat{m} \hat{n}}=-\Lambda_{\hat{n} \hat{m}} .
$$

The non-linear realization on the $\left(x_{m}, r\right)$ coordinates (2.7) may therefore be found from the relation (2.13), by identifying

$$
\begin{aligned}
& a^{m}=\Lambda_{-}^{m} ; \quad \lambda_{M}^{m n}=\Lambda^{m n} \\
& \lambda_{D}=\Lambda_{+}^{+}=-\Lambda_{-}^{-} ; \quad \Lambda_{K}^{m}=\Lambda_{+}^{m}=\frac{1}{2} \Lambda^{-m} .
\end{aligned}
$$

The above construction makes it simple to find an $S O(p+1,2) \times S O(d-$ $p-1)$ invariant $(p+2)$-form field strength $d \mathcal{A}$ on $\mathcal{M}_{d}$. We start from the following $(p+2)$-form on $\mathcal{S}_{d+2}$ : (with $\varepsilon_{01 \ldots p+-}=\frac{1}{2}$, an anti-density as required to be constant)

$$
\Omega_{p+2}=\frac{p+1}{(p+2) !(w R)^{2}} \varepsilon_{\hat{m}_{0} \ldots \hat{m}_{p+2}} X^{\hat{m}_{0}} d X^{\hat{m}_{1}} d X^{\hat{m}_{2}} \ldots d X^{\hat{m}_{p+2}} .
$$

This is manifestly $S O(p+1,2)$ invariant and trivially $S O(d-p-1)$ invariant. When restricted to $\mathcal{M}_{d}$ through (2.11), it becomes proportional to the volume form of $a d S_{p+2}$, which is closed on the constraint surface. In fact $\Omega_{p+2}=d \mathcal{A}$ on this hypersurface, where

$$
\begin{aligned}
\mathcal{A} & =d x^{0} \ldots d x^{p} \phi^{p+1} \\
& =d x^{0} \ldots d x^{p}\left(\frac{r}{R}\right)^{\frac{p+1}{w}} .
\end{aligned}
$$

For later convenience, we combine on $\mathcal{M}_{d}$ the horospherical coordinate $r$ and the hyperspherical coordinates $\theta_{i}$ into $d-p-1$ cartesian coordinates $X^{m^{\prime}}$. The following relations hold (with $i=1, \ldots, d-p-2$ ):

$$
\begin{aligned}
& r^{2}=X^{m^{\prime}} \delta_{m^{\prime} n^{\prime}} X^{n^{\prime}}, \\
& \theta_{i}=\arctan \left[\frac{\left(\sum_{k^{\prime}=i+p+1}^{d-1}\left(X^{k^{\prime}}\right)^{2}\right)^{1 / 2}}{X^{(p+i)^{\prime}}}\right] .
\end{aligned}
$$

With the above choice of background and in these coordinates the action (2.1) becomes

$$
S=-\int_{W} d^{p+1} \sigma\left[\sqrt{-\operatorname{det} g_{\mu \nu}}+\left(\frac{r}{R}\right)^{\frac{p+1}{w}}\left(\varepsilon^{\mu_{0} \ldots \mu_{p}} \partial_{\mu_{0}} x^{0} \ldots \partial_{\mu_{p}} x^{p}\right)\right],
$$


where

$$
g_{\mu \nu}=\left(\frac{r}{R}\right)^{\frac{2}{w}} \partial_{\mu} x^{m} \partial_{\nu} x^{n} \eta_{m n}+\left(\frac{R}{r}\right)^{2} \partial_{\mu} X^{m^{\prime}} \partial_{\nu} X^{n^{\prime}} \delta_{m^{\prime} n^{\prime}} .
$$

The $S O(p+1,2) \times S O(d-p-1)$ action follows, as we have essentially seen above, from its equivalence to the manifestly $S O(p+1,2) \times S O(d-p-1)$ action

$$
\hat{I}=\hat{I}_{D}+\hat{I}_{W Z}+\hat{I}_{L M}
$$

where $\hat{I}_{D}$ is the standard Dirac $p$-brane action with a metric induced from the flat metric on $\mathcal{S}_{d+2}$ :

$$
\hat{I}_{\mathrm{D}}=-\int_{W} d^{p+1} \sigma \sqrt{-\operatorname{det} \hat{g}_{\mu \nu}} ; \quad \hat{g}_{\mu \nu}=\partial_{\mu} X^{\hat{M}} \partial_{\nu} X^{\hat{N}} \eta_{\hat{M} \hat{N}}
$$

The Wess-Zumino term is

$$
\hat{I}_{W Z}=\int_{B} \Omega_{p+2}^{B}
$$

where $\Omega_{p+2}^{B}$ is the pullback of $\Omega_{p+2}$ in 2.17$)$ to a $(p+2)$-dimensional manifold $B$ with boundary $\partial B=W$. The 'Lagrange multiplier' action $\hat{I}_{L M}$ imposes the constraints $T_{1}=T_{2}=0$ via Lagrange multipliers,

$$
\hat{I}_{L M}=\int_{B} d^{p+2} \hat{\sigma}\left(\lambda^{1} T_{1}+\lambda^{2} T_{2}\right)
$$

Upon use of the constraints $T_{1}=T_{2}=0$ we are restricted in target space to $\mathcal{M}_{d}$, and we may use $\Omega_{p+2}=d \mathcal{A}$. The action $\hat{I}$ thus reduces to 2.20 ).

In the following section we shall see how this reformulation of the standard $p$-brane action applies to various cases of interest in M-theory and superstring theory. First we consider its symmetries and gauge fixing to a conformal action.

\subsection{Symmetries and gauge fixing}

The action (2.20) is invariant under local world-volume diffeomorphisms $\delta_{l d}(\eta)$ and under the rigid symmetries determined by the Killing equation, i.e. the $a d S$ symmetries $\delta_{a d S}(\xi)$ and sphere isometries. We shall fix the local gauge invariance by the 'physical', or 'static', gauge condition

$$
x^{\mu}(\sigma) \equiv \delta^{\mu}{ }_{m} x^{m}(\sigma)=\sigma^{\mu} .
$$


The gauge-fixed action is then

$$
S_{g . f}=\left.S\right|_{x=\sigma} .
$$

The gauge-fixing breaks not only the local diffeomorphisms (as it is designed to do) but also the rigid adS symmetry. However, the action will still be invariant under some combination of $a d S$ symmetry plus 'compensating' local diffeomorphisms $\delta_{l d}(\eta)$, for a particular 'rigid' parameter $\eta(\sigma)$ defined by the requirement

$$
\left.\left[\delta_{a d S}(\xi)+\delta_{l d}(\eta(\sigma))\right] x^{\mu}\right|_{x=\sigma}=0,
$$

which yields

$$
\eta^{\mu}(\sigma)=\hat{\xi}^{\mu}(\sigma, r(\sigma)) .
$$

We thus find that the gauge-fixed action (2.27) is invariant under the following conformal transformations

$$
\begin{aligned}
\delta_{C} X^{m^{\prime}} & =\hat{\xi}^{\mu}(\sigma, r(\sigma)) \partial_{\mu} X^{m^{\prime}}+w \Lambda_{D}(\sigma) X^{m^{\prime}} \\
& =\xi^{\mu}(\sigma) \partial_{\mu} X^{m^{\prime}}+w \Lambda_{D}(\sigma) X^{m^{\prime}}+(w R)^{2} \Lambda_{K}^{\mu}\left(\frac{R}{r}\right)^{\frac{2}{w}} \partial_{\mu} X^{m^{\prime}},
\end{aligned}
$$

which are of the general form (2.4) in [6], identifying $w$ as the Weyl weight of $X^{m^{\prime}}$, and the special conformal transformations acquire an extra part

$$
k_{\mu} X^{m^{\prime}}=(w R)^{2}\left(\frac{R}{r}\right)^{2 / w} \partial_{\mu} X^{m^{\prime}} .
$$

For completeness we give the $S O(d-p-1)$ 'R-symmetry' acting linearly on the $X^{m^{\prime}}$ as

$$
\delta_{R} X^{m^{\prime}}=-\Lambda_{n^{\prime}}^{m^{\prime}} X^{n^{\prime}}, \quad \Lambda_{m^{\prime} n^{\prime}}=-\Lambda_{n^{\prime} m^{\prime}} .
$$

In arriving at this result we have implicitly assumed that the gauge condition $x^{\mu}=\sigma^{\mu}$ is valid globally, rather than just locally. This assumption restricts the possible worldvolume field configurations to some set that includes an infinite static brane aligned with the cartesian $x^{\mu}$ axes. The worldvolume of this infinite static brane will be identified as the Minkowski vacuum of the gauge-fixed action. For this worldvolume vacuum to be stable it must be a solution of the worldvolume field equations (the 'branewave' equations) but it is only in very special backgrounds that such an infinite static brane solution exists. In the backgrounds considered here it was once thought that an infinite static brane could solve the branewave equations only if placed at 
the adS boundary at $r=\infty$ [2]. This is indeed a solution but not the only one: in the coordinates used here the 'vacuum' configuration

$$
x^{\mu}=\sigma^{\mu}, \quad r=\langle r\rangle,
$$

with constant position on the $(d-p-2)$-sphere, is a solution for any value of $\langle r\rangle$.

\section{M2, D3, M5 and D1+D5 conformal field theories}

We now have most of the ingredients needed to demonstrate the conformal invariance of the bosonic M2, D3, M5 and D1+D5 brane actions in their own near-horizon backgrounds. The space-time metric and the $(p+1)$-form of the brane configuration with $1 / 2$ of unbroken supersymmetry are described in terms of a harmonic function

$$
H_{\text {brane }}=1+\left(\frac{R}{r}\right)^{\frac{p+1}{w}}, \quad r^{2}=X^{m^{\prime}} \delta_{m^{\prime} n^{\prime}} X^{n^{\prime}},
$$

which solves the Laplace equation in the space of $d-p-1$ transverse directions $X^{m^{\prime}}$ to the brane. The metric is $\left(m=0,1, \ldots, p ; m^{\prime}=p+1, \ldots, d-1\right)$ :

$$
d s_{\text {brane }}^{2}=H_{\text {brane }}^{-\frac{2}{p+1}} d x^{m} \eta_{m n} d x^{n}+H_{\text {brane }}^{\frac{2 w}{p+1}} d X^{m^{\prime}} \delta_{m^{\prime} n^{\prime}} d X^{n^{\prime}} .
$$

The brane space-time is asymptotically flat at $r \rightarrow \infty$. The horizon of the brane in these isotropic coordinates is at $r \rightarrow 0$. Very close to the horizon at $r \rightarrow 0$ the first term in the harmonic function (3.1) can be neglected comparative to the second term. This gives a near-horizon geometry as in

(2.6) and a $(p+1)$-form as in (2.18) [3]. It has been pointed out in [7] that one can interpret the parameter $R$ for each of the configurations above as follows. For the M2 brane $R^{6}=2^{5} \pi^{2} N l_{P}^{6}$, for the D3 brane $R^{4}=4 \pi g N \alpha^{\prime 2}$, and for M5 $R^{3}=\pi N l_{P}^{3}$ and $N$ can be interpreted as the number of parallel branes. It is possible therefore to think of the $a d S_{p+2} \times S^{d-p-2}$ geometry as coming either as a result of considering a small $r$ (near horizon) approximation or a large $N$ (many branes with unit charges) approximation. Note also that since the curvature near the horizon depends on $\mathrm{N}$ as a negative power, large $N$ is required for supergravity to be valid. An additional interpretation 
was suggested in [15, 17, 21], where a special duality transformation was introduced to remove the constant from the harmonic function. It is this metric (2.6), which gives the target space metric $\mathcal{G}$ that is pulled back to the brane worldvolume to lead to the metric $g_{\mu \nu}$ used in section 2 .

The above formalism with the relation (2.4) can be applied in all the cases in table 1. The first three cases have in common the feature that $w$ is the

\begin{tabular}{||l|c|c|c||}
\hline & d & p & w \\
\hline M2 & 11 & 2 & $1 / 2$ \\
M5 & 11 & 5 & 2 \\
D3 & 10 & 3 & 1 \\
Self-dual string & 6 & 1 & 1 \\
Magnetic string & 5 & 1 & 2 \\
Tangerlini black hole & 5 & 0 & $1 / 2$ \\
Reissner-Nordström black hole & 4 & 0 & 1 \\
\hline
\end{tabular}

Table 1: Solutions with an $a d S_{p+2} \times S^{d-p-2}$ geometry.

canonical Weyl weight of a world-volume scalar field, i.e.

$$
w=\frac{1}{2}(p-1) \quad(\mathrm{M} 2, \mathrm{D} 3, \mathrm{M} 5) .
$$

The other ones can be obtained as intersecting branes and have as such been mentioned in 116, 17, 18]. Then the resulting geometry has as extra factors Euclidean manifolds. Apart from M2, D3 and M5, we shall treat here the $d=6$ case as the intersection 'D1+D5'. The black hole cases have some interesting special features which will be discussed in a separate article [22].

The world-volume actions are in all those cases similar to those treated in section 2, but we will need modifications of the Wess-Zumino (WZ) terms, and additions due to the presence of a world-volume vector for D3 and a world-volume antisymmetric tensor for M5.

\subsection{M2 brane}

The near-horizon background of the M2 brane is the $a d S_{4} \times S^{7}$ solution as mentioned before, i.e. (2.6) and (2.18) with $d=11, p=2$ and $w=\frac{1}{2}$. The results found there apply without change. The gauge-fixed M2 brane action 
can be expanded in a power series in $R$, which is appropriate in the small velocities approximation. The $R$-independent term is just a free conformal field theory so $R$ is the coupling constant, as pointed out in $[7,8]$. Details of this expansion can be found in Appendix Q. The action up to fourth order in derivatives of fields is

$$
\begin{aligned}
S_{\mathrm{M} 2}=-\int d^{3} \sigma & \left\{\frac{1}{2} \partial^{\bar{\mu}} X^{m^{\prime}} \partial_{\bar{\mu}} X_{m^{\prime}}\right. \\
& -\frac{1}{4}\left(\frac{R}{r}\right)^{6}\left[\partial^{\bar{\mu}} X^{m^{\prime}} \partial_{\bar{\nu}} X_{m^{\prime}} \partial^{\bar{\nu}} X^{n^{\prime}} \partial_{\bar{\mu}} X_{n^{\prime}}-\frac{1}{2}\left(\partial^{\bar{\mu}} X^{m^{\prime}} \partial_{\bar{\mu}} X_{m^{\prime}}\right)^{2}\right] \\
& +\ldots\} .
\end{aligned}
$$

The indices $\bar{\mu}$ are barred to indicate contraction with the flat metric $\eta_{\bar{\mu} \bar{\nu}}$. Note that the truncation to $R=0$ preserves the conformal symmetry and gives a free scalar multiplet in 3 dimensions.

\subsection{D3 brane}

The near-horizon geometry of the D3 brane is the $a d S_{5} \times S^{5}$ IIB supergravity solution $(w=1)$

$$
\begin{aligned}
d s^{2}= & \left(\frac{r}{R}\right)^{2} d x^{m} d x^{n} \eta_{m n}+\left(\frac{R}{r}\right)^{2}\left[d r^{2}+r^{2} d \Omega^{2}\right] \\
\mathcal{A}_{4}= & \left(\frac{r}{R}\right)^{4} d x^{0} d x^{1} d x^{2} d x^{3} \\
& +4 R^{4} \sin ^{4} \theta_{1} \sin ^{3} \theta_{2} \sin ^{2} \theta_{3} \sin \theta_{4} \theta_{5} d \theta_{1} d \theta_{2} d \theta_{3} d \theta_{4} .
\end{aligned}
$$

Note that the latter is not unique, e.g. the last term could also be chosen as

$$
4 R^{4} \sin ^{4} \theta_{1} \sin ^{3} \theta_{2} \sin ^{2} \theta_{3} \cos \theta_{4} d \theta_{1} d \theta_{2} d \theta_{3} d \theta_{5} .
$$

This is similar to, but not quite the same as, the $d=10, p=3$ case of the previous section. The difference arises because the 5 -form field strength of $\mathcal{A}_{4}$ is now required to be self-dual. The WZ term must therefore be changed accordingly. We can accomplish this in a manifestly $S O(4,2) \times S O(5)$ invariant way as follows. We introduce the additional manifestly $S O(6)$-invariant 5 -form

$$
\tilde{\Omega}_{5}=\frac{4}{5 ! R^{2}} \varepsilon_{\hat{m}_{0}^{\prime} \hat{m}_{1}^{\prime} \ldots \hat{m}_{5}^{\prime}} X^{\hat{m}_{0}^{\prime}} d X^{\hat{m}_{1}^{\prime}} \ldots d X^{\hat{m}_{5}^{\prime}}
$$


On the hypersurface $T_{2}=0$ this is proportional to the volume form on $S^{5}$, so it is the hodge dual ${ }^{3}$ in $\mathcal{M}_{d}$ of the restriction to the hypersurface $T_{1}=0$ of $\Omega_{5}$. It follows that the 5 -form

$$
\Omega_{\mathrm{D} 3}=\Omega_{5}+\tilde{\Omega}_{5}
$$

is the self-dual field strength of $\mathcal{A}_{4}$ on the constraint surface. The D3 WZ term is therefore obtained from the one of the previous section by the replacement $\Omega_{5} \rightarrow \Omega_{D 3}$. A modification is also needed to the 'Dirac' action to incorporate the world-volume Born-Infeld 1-form $V$. We take

$$
\hat{I}_{D} \rightarrow I_{D B I} \equiv-\int d^{4} \sigma \sqrt{-\operatorname{det}(g+F)},
$$

where $g$ is the metric induced from the flat metric on $\mathcal{S}_{d+2}$ and $F=d V$, which is inert under isometries of $g$ (note that the NS 2-form vanishes in the background that we consider). The gauge-fixed action is then invariant under $(2.30),(2.32)$ and

$$
\begin{aligned}
\delta_{C} F_{\mu \nu}= & \hat{\xi}^{\rho}(\sigma, r) \partial_{\rho} F_{\mu \nu}-2 \partial_{[\mu} \xi^{\rho}(\sigma, r) F_{\nu] \rho} \\
= & \xi^{\rho}(\sigma) \partial_{\rho} F_{\mu \nu}-2 \Lambda_{M}(\sigma)^{\rho}{ }_{[\mu} F_{\nu] \rho}+2 \Lambda_{D}(\sigma) F_{\mu \nu} \\
& +R^{2} \Lambda_{K}^{\rho}\left[\left(\frac{R}{r}\right)^{2} \partial_{\rho} F_{\mu \nu}-2 \partial_{[\mu}\left(\frac{R}{r}\right)^{2} F_{\nu] \rho}\right] .
\end{aligned}
$$

This is again of the general form of conformal transformations with e.g. on the vector $V_{\mu}$ the extra transformation

$$
k_{\nu} V_{\mu}=R^{2}\left(\frac{R}{r}\right)^{2} \partial_{\nu} V_{\mu}+R^{2} \partial_{\mu}\left(\frac{R}{r}\right)^{2} V_{\nu} .
$$

This drops out for $R=0$ and in that limit we get the free action of the bosonic part of a $N=4$ vector multiplet in 4 dimensions.

The D3 brane gives a 4-dimensional interacting conformal theory of a vector supermultiplet which can again be expanded as a power series in $R$ or, equivalently, in powers of derivatives of fields. The expansion to fourth order in derivatives is

$$
S_{\mathrm{D} 3}=-\int d^{4} \sigma\left\{\frac{1}{2} \partial^{\bar{\mu}} X^{m^{\prime}} \partial_{\bar{\mu}} X_{m^{\prime}}+\frac{1}{4} F^{\bar{\mu} \bar{\nu}} F_{\bar{\mu} \bar{\nu}}\right.
$$

\footnotetext{
${ }^{3}$ The proportionality constant between $\Omega_{5}$ and the volumeform on $a d S_{5}$ is the same as between $\tilde{\Omega}_{5}$ and the volume form on $S^{5}$.
} 


$$
\begin{aligned}
& -\frac{1}{4}\left(\frac{R}{r}\right)^{4}\left[\partial^{\bar{\mu}} X^{m^{\prime}} \partial_{\bar{\nu}} X_{m^{\prime}} \partial^{\bar{\nu}} X^{n^{\prime}} \partial_{\bar{\mu}} X_{n^{\prime}}-\frac{1}{2}\left(\partial^{\bar{\mu}} X^{m^{\prime}} \partial_{\bar{\mu}} X_{m^{\prime}}\right)^{2}\right] \\
& -\frac{1}{8}\left(\frac{R}{r}\right)^{4}\left[F^{\bar{\mu} \bar{\nu}} F_{\bar{\nu} \bar{\rho}} F^{\bar{\rho} \bar{\sigma}} F_{\bar{\sigma} \bar{\mu}}-\frac{1}{4}\left(F^{\bar{\mu} \bar{\nu}} F_{\bar{\mu} \bar{\nu}}\right)^{2}\right] \\
& +\frac{1}{2}\left(\frac{R}{r}\right)^{4}\left[\partial^{\bar{\mu}} X^{m^{\prime}} \partial_{\bar{\nu}} X_{m^{\prime}} F^{\bar{\nu} \bar{\rho}} F_{\bar{\rho} \bar{\mu}}+\frac{1}{4} \partial^{\bar{\mu}} X^{m^{\prime}} \partial_{\bar{\mu}} X_{m^{\prime}} F^{\bar{\nu} \bar{\rho}} F_{\bar{\nu} \bar{\rho}}\right] \\
& +4 R^{4} \sin ^{4} \theta_{1} \sin ^{3} \theta_{2} \sin ^{2} \theta_{3} \sin \theta_{4} \theta_{5} \varepsilon^{\bar{\mu} \bar{\nu} \bar{\rho} \bar{\sigma}} \partial_{\bar{\mu}} \theta_{1} \partial_{\bar{\nu}} \theta_{2} \partial_{\bar{\rho}} \theta_{3} \partial_{\bar{\sigma}} \theta_{4} \\
& +\ldots\},
\end{aligned}
$$

where $\left(X^{m^{\prime}}, r, \theta_{i}\right)$ are related through $(2.19)$.

\subsection{M5 brane}

The near-horizon geometry of the M5 brane f $^{\text {is }}$ the $a d S_{7} \times S^{4}$ solution of $\mathrm{D}=11$ supergravity $(w=2)$

$$
\begin{aligned}
d s^{2} & =\left(\frac{r}{R}\right) d x^{m} d x^{n} \eta_{m n}+\left(\frac{R}{r}\right)^{2}\left[d r^{2}+d \Omega^{2}\right] \\
\mathcal{A}_{3} & =-3 R^{3} \sin ^{3} \theta_{1} \sin ^{2} \theta_{2} \sin \theta_{3} \theta_{4} d \theta_{1} d \theta_{2} d \theta_{3} .
\end{aligned}
$$

In order to cast this into the $d=11, p=5$ case of the previous section we need to give the solution for $\mathcal{A}_{3}$ in terms of its magnetic dual potential $\mathcal{A}_{6}$. In general, the two are related by $d \mathcal{A}_{6}={ }^{\star} d \mathcal{A}_{3}+\mathcal{A}_{3} \wedge d \mathcal{A}_{3}$, but the second term on the right hand side vanishes for the particular $\mathcal{A}_{3}$ of the above solution. The dual 6-form potential therefore satisfies $d \mathcal{A}_{6}={ }^{\star} d \mathcal{A}_{3}$. We can choose it be

$$
\mathcal{A}_{6}=\left(\frac{r}{R}\right)^{3} d x^{0} d x^{1} \ldots d x^{5} .
$$

We see from this that the $a d S_{7} \times S^{4}$ background is of the form assumed in section 2. The WZ term includes an integral over the 6-form potential, which can be dealt with as explained previously, via the introduction of the 7 -form $\Omega_{7}$.

However, there is another contribution to the WZ term involving the world-volume 3 -form field strength $\mathcal{H}$, which we will introduce on $B$, subject to the Bianchi identity

$$
d \mathcal{H}+d \mathcal{A}_{3}^{B}=0 .
$$

\footnotetext{
${ }^{4}$ For M5 we will use the formulation of [23], following the notation and conventions of [6].
} 
We shall consider $\mathcal{H}$ to be an independent 3 -form field and we shall introduce its Bianchi 'identity' by means of a 3-form Lagrange multiplier $K_{3}$. Specifically, we add to the action the term

$$
I_{L M}^{\prime}=\int_{B} K_{3} \wedge\left(d \mathcal{H}+\tilde{\Omega}_{4}^{B}\right)
$$

where $\tilde{\Omega}_{4}^{B}$ is the pullback to the space $B$ of

$$
\tilde{\Omega}_{4}=\frac{3}{4 ! R^{2}} \varepsilon_{\hat{n}^{\prime} \hat{m}_{1}^{\prime} \ldots \hat{m}_{4}^{\prime}} X^{\hat{n}^{\prime}} d X^{\hat{m}_{1}^{\prime}} \ldots d X^{\hat{m}_{4}^{\prime}} .
$$

The Lagrange multiplier $K_{3}$ imposes the constraint $d \mathcal{H}=-\tilde{\Omega}_{4}^{B}$, which requires $\tilde{\Omega}_{4}^{B}$ to be closed. It is sufficient that this is true on the constraint surface $T_{1}=T_{2}=0$, which it is because on this surface $\tilde{\Omega}_{4}$ is the hodge dual of $\Omega_{7}$ (see (2.17)) and hence equal to $d \mathcal{A}_{3}$. The M5 brane WZ term can now be written in a manifestly $S O(6,2) \times S O(5)$ invariant way as the integral over $B$ of the 7 -form

$$
\Omega_{M 5}=\Omega_{7}^{B}-\frac{1}{2} \mathcal{H} \wedge \tilde{\Omega}_{4}^{B} .
$$

On the constraint surface (and imposing the constraint $d \mathcal{H}=\tilde{\Omega}_{4}^{B}$ ) we have

$$
\begin{aligned}
& \Omega_{7}=d \mathcal{A}_{6} ; \quad \tilde{\Omega}_{4}^{B}=d \mathcal{A}_{3}=-d \mathcal{H} ; \quad \mathcal{A}_{3} \wedge d \mathcal{A}_{3}=0 \\
& \Rightarrow \quad \Omega_{M 5}=d\left(\mathcal{A}_{6}^{B}+\frac{1}{2} \mathcal{H} \wedge \mathcal{A}_{3}^{B}\right),
\end{aligned}
$$

so that the WZ term reduces, locally, to an integral over the 6-dimensional boundary of $B$. It is, in fact, just the WZ term of the M5 brane action of [23]. The $S O(6,2) \times S O(5)$ invariance is now manifest if we take $\mathcal{H}$, and the Lagrange multiplier $K_{3}$ to be invariant (as we may do since their transformations must be specified independently of those of all other fields). A modification to the 'Dirac' action relative to the previous section is also required. To obtain it we proceed as follows: we define [23]

$$
\begin{aligned}
u_{\mu} & =\partial_{\mu} a, \quad v_{\mu}=\frac{u_{\mu}}{\sqrt{u^{2}}}, \quad u^{2}=u \hat{g}^{\mu \nu} u_{\nu}, \\
\mathcal{H}_{\mu \nu \rho}^{*} & =\frac{\sqrt{-\operatorname{det} \hat{g}}}{6} \varepsilon_{\mu \nu \rho \sigma \lambda \eta} \hat{g}^{\sigma \tau} \hat{g}^{\lambda \kappa} \hat{g}^{\eta \phi} \mathcal{H}_{\tau \kappa \phi}, \\
\mathcal{H}_{\mu \nu} & =v^{\rho} \mathcal{H}_{\mu \nu \rho}, \quad \mathcal{H}_{\mu \nu}^{*}=v^{\rho} \mathcal{H}_{\mu \nu \rho}^{*},
\end{aligned}
$$

where $a$ is the 'PST' auxiliary field, and $\hat{g}$ is the induced world-volume metric from the flat metric in $\mathcal{S}_{d+2}$. 
We must then make the replacement

$$
\hat{I}_{D} \rightarrow \hat{I}_{B L N P S T} \equiv-\int d^{6} \sigma\left[\sqrt{-\operatorname{det}\left(\hat{g}+i \mathcal{H}^{*}\right)}+\frac{1}{4} \sqrt{-\operatorname{det} \hat{g}} \mathcal{H}^{* \mu \nu} \mathcal{H}_{\mu \nu}\right]
$$

This is again manifestly $S O(6,2) \times S O(5)$ invariant. The Bianchi identity can be solved for a two form potential $B_{\mu \nu}$, i.e.

$$
\mathcal{H}=d B-\mathcal{A}_{3}^{B}
$$

Note that $\tilde{\Omega}_{4}$ is invariant under $S O(5)$, but an explicit solution for $\mathcal{A}_{3}$ of $\tilde{\Omega}_{4}=d \mathcal{A}_{3}$ is not invariant. E.g. the one we gave in (3.12) is not invariant under a shift of $\theta_{4}$. But we should have that

$$
\delta_{R}(\Lambda) \mathcal{A}_{3}=d C_{2}(\Lambda)
$$

for some 2-form $C_{2}$. The requirement that $\mathcal{H}$ is invariant under the background isometries forces the potential $B$ to transform under $S O(5)$ as

$$
\delta_{R}(\Lambda) B=C_{2}(\Lambda)
$$

(up to a gauge transformation). Therefore the algebra of isometries could acquire central charges. Indeed, from descent equations

$$
\delta_{R}\left(\Lambda^{\prime}\right) C_{2}(\Lambda)-\delta_{R}(\Lambda) C_{2}\left(\Lambda^{\prime}\right)=C_{2}\left(\left[\Lambda, \Lambda^{\prime}\right]\right)+d C_{1}\left(\Lambda, \Lambda^{\prime}\right)
$$

It is possible that $C_{1}$ vanishes, and with the choice of $\mathcal{A}_{3}$ in (3.12) partial calculations indicate that a $C_{2}$ exists with vanishing $C_{1}$.

It is straightforward to compute the conformal transformations from the decomposition rule $(2.29)$. The transformation of the fields appearing in the action, can be deduced from the following. Besides (2.30) and (2.32), we have

$$
\begin{aligned}
\delta_{C} a= & \hat{\xi}^{\mu}(\sigma, r) \partial_{\mu} a=\xi^{\mu}(\sigma) \partial_{\mu} a+4 R^{2} \Lambda_{K}^{\mu}\left(\frac{R}{r}\right) \partial_{\mu} a \\
\delta_{C} B_{\mu \nu}= & \hat{\xi}^{\rho}(\sigma, r) \partial_{\rho} B_{\mu \nu}-2 \partial_{[\mu} \xi^{\rho}(\sigma, r) B_{\nu] \rho} \\
= & \xi^{\rho}(\sigma) \partial_{\rho} B_{\mu \nu}-2 \Lambda_{M}(\sigma)^{\rho}{ }_{[\mu} B_{\nu] \rho}+2 \Lambda_{D}(\sigma) B_{\mu \nu} \\
& +R^{2}\left[\left(\frac{R}{r}\right)^{2} \partial_{\rho} B_{\mu \nu}-2 \partial_{[\mu}\left(\frac{R}{r}\right)^{2} B_{\nu] \rho}\right] .
\end{aligned}
$$


The M5 brane gives a 6-dimensional conformal theory of a tensor supermultiplet. The bosonic action in the same approximation as before is

$$
\begin{aligned}
S_{\mathrm{M} 5}=-\int d^{6} \sigma & \left\{\frac{1}{2} \partial^{\bar{\mu}} X^{m^{\prime}} \partial_{\bar{\mu}} X_{m^{\prime}}+\frac{1}{2} \mathcal{H}^{* \bar{\mu} \bar{\nu}} \mathcal{H}_{\bar{\mu} \bar{\nu}}^{-}\right. \\
& -\frac{1}{4}\left(\frac{R}{r}\right)^{3}\left[\partial^{\bar{\mu}} X^{m^{\prime}} \partial_{\bar{\nu}} X_{m^{\prime}} \partial^{\bar{\nu}} X^{n^{\prime}} \partial_{\bar{\mu}} X_{n^{\prime}}-\frac{1}{2}\left(\partial^{\bar{\mu}} X^{m^{\prime}} \partial_{\bar{\mu}} X_{m^{\prime}}\right)^{2}\right] \\
& -\frac{1}{8}\left(\frac{R}{r}\right)^{3}\left[\mathcal{H}^{* \bar{\mu} \bar{\nu}} \mathcal{H}_{\bar{\nu} \bar{\rho}}^{*} \mathcal{H}^{* \bar{\rho} \bar{\sigma}} \mathcal{H}_{\bar{\sigma} \bar{\mu}}^{*}-\frac{1}{4}\left(\mathcal{H}^{* \bar{\mu} \bar{\nu}} \mathcal{H}_{\bar{\mu} \bar{\nu}}^{*}\right)^{2}\right] \\
& +\frac{1}{2}\left(\frac{R}{r}\right)^{3}\left[\partial^{\bar{\mu}} X^{m^{\prime}} \partial_{\bar{\nu}} X_{m^{\prime}} \mathcal{H}^{* \bar{\nu} \bar{\rho}} \mathcal{H}_{\bar{\rho} \bar{\mu}}^{*}+\frac{1}{4} \partial^{\bar{\mu}} X^{m^{\prime}} \partial_{\bar{\mu}} X_{m^{\prime}} \mathcal{H}^{* \bar{\nu} \bar{\rho}} \mathcal{H}_{\bar{\nu} \bar{\rho}}^{*}\right] \\
& +\frac{1}{2}\left(\frac{R}{r}\right)^{3}\left[v_{\bar{\rho}} \partial^{\bar{\rho}} X^{m^{\prime}} \partial^{\bar{\sigma}} X_{m^{\prime}} v_{\bar{\sigma}} \mathcal{H}^{* \bar{\mu} \bar{\nu}} \mathcal{H}_{\bar{\mu} \bar{\nu}}^{-}\right. \\
& \left.-\frac{1}{2} v_{\bar{\rho}} \partial^{\bar{\rho}} X^{m^{\prime}} \partial^{\bar{\sigma}} X_{m^{\prime}} \mathcal{H}_{\bar{\sigma} \bar{\mu} \bar{\nu}} \mathcal{H}^{* \bar{\mu} \bar{\nu}}\right] \\
& +\frac{1}{4} R^{2} \sin ^{3} \theta_{1} \sin ^{2} \theta_{2} \sin \theta_{3} \theta_{4} \varepsilon^{\bar{\mu} \bar{\nu} \bar{\rho} \bar{\alpha} \bar{\tau}} \mathcal{H}_{\bar{\mu} \bar{\nu} \bar{\rho}} \partial_{\bar{\sigma}} \theta_{1} \partial_{\bar{\lambda}} \theta_{2} \partial_{\bar{\tau}} \theta_{3} \\
& +\ldots\} .
\end{aligned}
$$

\subsection{D1+D5}

The self-dual string near-horizon geometry is $a d S_{3} \times S^{3}$ which is very similar to the $a d S_{3} \times S^{3} \times E^{4}$ near-horizon geometry of the solution representing a D1-brane embedded within a D5-brane 9 . In IIB supergravity in the string frame, this solution is given by

$$
\begin{aligned}
d s_{\text {brane }}^{2}= & \left(H_{1} H_{5}\right)^{-\frac{1}{2}}\left(-\left(d x^{0}\right)^{2}+\left(d x^{5}\right)^{2}\right)+H_{1}^{\frac{1}{2}} H_{5}^{-\frac{1}{2}}\left(\left(d x^{1}\right)^{2}+\ldots+\left(d x^{4}\right)^{2}\right) \\
& +\left(H_{1} H_{5}\right)^{\frac{1}{2}}\left(\left(d x^{6}\right)^{2}+\ldots+\left(d x^{9}\right)^{2}\right) \\
\mathcal{F}_{3}= & \Omega_{3}+\tilde{\Omega}_{3} \\
\Omega_{3}= & d x^{0} d x^{5} d H_{1}^{-1} ; \quad \star \tilde{\Omega}_{3}=d x^{0} d x^{1} d x^{2} d x^{3} d x^{4} d x^{5} d H_{5}^{-1} \\
H_{1}= & 1+\left(\frac{c_{1}}{r}\right)^{2} ; \quad H_{5}=1+\left(\frac{c_{5}}{r}\right)^{2}, \quad e^{-2 \phi}=H_{5} H_{1}^{-1} .
\end{aligned}
$$

This solution was found in 24] by performing an S-duality rotation of NSNS $1+5$ solution [25]. For the case of the string+fivebrane solution in NS-

\footnotetext{
${ }^{5}$ The near horizon behaviour and enhancement of supersymmetry of various intersecting and overlapping branes was studied in [16] and [17. We derive the enhancement of supersymmetry for D1+D5 in Appendix B.
} 
NS form it was shown that the string action in the conformal gauge in the near-horizon region reduces to the direct product of 2-dimensional conformal theories: $S L(2, R)$ and $S U(2)$ WZW theories and a free 5-torus theory. The relation between this conformal theory and the one we discuss here is still to be understood.

In what follows we will use $c_{1}=c_{5}=R$, i.e. $H_{1}=H_{5}$. Note that in the near-horizon regime, the solution assumes the form of $a d S_{3} \times S^{3} \times E^{4}$ $\left(r^{2}=\left(x^{6}\right)^{2}+\ldots+\left(x^{9}\right)^{2}\right)$.

$$
\begin{aligned}
d s^{2}= & \left(\frac{r}{R}\right)^{2}\left[-\left(d x^{0}\right)^{2}+\left(d x^{5}\right)^{2}\right]+\left(\frac{R}{r}\right)^{2}\left[d r^{2}+r^{2} d \Omega^{2}\right] \\
& +\left[\left(d x^{1}\right)^{2}+\ldots+\left(d x^{4}\right)^{2}\right] \\
\mathcal{A}_{2}= & \left(\frac{r}{R}\right)^{2} d x^{0} d x^{5}+2 R^{2} \sin ^{2} \theta_{1} \sin \theta_{2} \theta_{3} d \theta_{1} d \theta_{2} ; \quad e_{\text {hor }}^{-2 \phi}=1
\end{aligned}
$$

This solution is of the form $a d S_{3} \times S^{3} \times E^{4}$, i.e. the product of $E^{4}$ with the submanifold defined in section 2 for $d=6$ as the intersection of two hypersurfaces $T_{1}=T_{2}=0$ in a flat space of signature $(6,2)$. Equivalently, we may define it as a submanifold of space of signature $(10,2)$ subject to the same $(d=6)$ constraints (which are therefore independent of the $E^{4}$ coordinates).

Let $g$ be the metric on the worldsheet of a D-string induced from the flat metric on this $(10,2)$ space. Since the dilaton is a constant in our chosen background, the DBI part of the action is simply

$$
S_{D B I}=\int d^{2} \sigma \sqrt{-\operatorname{det}\left(g_{\mu \nu}+F_{\mu \nu}\right)},
$$

where $F=d V$ is the field strength of the Born-Infeld 1-form potential $V$ (which we take to be inert under isometries of the background). This action is manifestly $S O(2,2) \times S O(4)$ invariant (and also manifestly invariant under the $I S O(4)$ isometry group of $\left.E^{4}\right)$, which remains from the original $I S O(6,2)$ (or $I S O(10,2)$ ) symmetry by the existence of a hypersurface. It was shown in 26] that (3.29) in 2 dimensions has more symmetries. An infinite dimensional extension of the adS isometries (2.7), written collectively as $\delta X^{M}=h^{M}(X)$, is provided by

$$
\begin{aligned}
\delta X^{M} & =h^{M}(X) \lambda(\mathcal{F}) \\
\delta V_{\mu} & =-\lambda^{\prime}(\mathcal{F}) \sqrt{g} \epsilon_{\mu \nu} H^{\nu}\left(1+\mathcal{F}^{2}\right)
\end{aligned}
$$


where

$$
\begin{aligned}
H^{\mu} & =g^{\mu \nu}\left(\partial_{\nu} X^{M}\right) g_{M N} h^{N}(X) \\
F_{\mu \nu} & =\partial_{\mu} V_{\nu}-\partial_{\nu} V_{\mu}=\epsilon_{\mu \nu} F \quad \text { or } \quad F=-\frac{1}{2} \epsilon^{\mu \nu} F_{\mu \nu} \\
\mathcal{F} & =\frac{F}{\sqrt{g}} ; \quad g=\operatorname{det}\left|g_{\mu \nu}\right| .
\end{aligned}
$$

The arbitrary function $\lambda$ thus provides a sort of Kač-Moody extension of the isometry group. Note that this is not the Virasoro extension. The Virasoro Killing vector of two dimensions is not a Killing vector of the 3-dimensional adS metric (only its $S U(1,1)$ part is). We do not yet see an application of this extension, and will thus omit it henceforth.

The WZ term of the D-string is (in the above background) the integral of $d \mathcal{A}_{2}^{B}$, the pullback of $\mathcal{A}_{2}$, over a 3 -dimensional manifold $B$ having the string worldsheet as its boundary. The WZ term is therefore $S O(2,2) \times$ $S O(4)$ invariant if the 3 -form $d \mathcal{A}_{2}$ is. Note that $d \mathcal{A}_{2}$ is actually a 3 -form on $a d S_{3} \times S^{3}$. In this context it is self-dual and its $S O(2,2) \times S O(4)$ invariance may therefore be made manifest in the same way that we made manifest the $S U(2,2) \times S U(4)$ invariance of the D3 brane WZ term. Specifically, we begin by defining

$$
\Omega_{3}=\frac{1}{3 R^{2}} \varepsilon_{\hat{m} \hat{n} \hat{p} \hat{q}} X^{\hat{m}} d X^{\hat{n}} d X^{\hat{p}} d X^{\hat{q}}, \quad \tilde{\Omega}_{3}=\frac{1}{3 R^{2}} \varepsilon_{\hat{m}^{\prime} \hat{n}^{\prime} \hat{p}^{\prime} \hat{q}^{\prime}} X^{\hat{m}^{\prime}} d X^{\hat{n}^{\prime}} d X^{\hat{p}^{\prime}} d X^{\hat{q}^{\prime}}
$$

where $X^{\hat{m}}$ are coordinates on the $(2+2)$ dimensional space in which $a d S_{3}$ is defined as the hypersurface $T_{1}=0$, and $X^{\hat{m}^{\prime}}$ are the coordinates on the 4-dimensional space in which $S^{3}$ is defined as the hypersurface $T_{2}=0$. We then take the WZ term to be the integral over $B$ of the 3 -form

$$
\Omega_{D 1}=\Omega_{3}+\tilde{\Omega}_{3}
$$

On the constraint hypersurface this reduces to $d \mathcal{A}_{2}$ of (3.28). Thus, the addition to the action of a Lagrange multiplier term for the constraints yields a manifestly $S O(2,2) \times S O(4)$ form of the D-string action in the $\mathrm{D} 1+\mathrm{D} 5$ background. The gauge-fixed non-linear action,

$$
S_{D 1}=-\int d^{2} \sigma\left(\frac{r}{R}\right)^{2}(\sqrt{-\operatorname{det}(g+F)}-1)+2 R^{2} \sin ^{2} \theta_{1} \sin \theta_{2} \theta_{3} \varepsilon^{\mu \nu} \partial_{\mu} \theta_{1} \partial_{\nu} \theta_{2}
$$


where

$$
g_{\mu \nu}=\eta_{\mu \nu}+\left(\frac{R}{r}\right)^{4} \partial_{\mu} X^{m^{\prime}} \partial_{\nu} X_{m^{\prime}}+\left(\frac{R}{r}\right)^{2} \partial_{\mu} X^{\tilde{m}} \partial_{\nu} X_{\tilde{m}}
$$

and $X^{\tilde{m}}$ are the coordinates of $E^{4}$, is invariant under the conformal transformations given in (2.30) with $w=1$ for $X^{m^{\prime}}$ and with $w=0$ for $X^{\tilde{m}}$, and for the vector under (3.9).

\section{Discussion}

M-theory and string theory $p$-brane solutions with non-singular Killing horizons are typically asymptotic near the horizon to spaces of the form $a d S_{p+2} \times$ $S^{d-p-2}$. Examples are the M2 and M5 branes $(d=11)$ and the IIB D3 brane $(d=10)$. In these cases we have shown that a test M2, D3 or M5 brane in its own near-horizon geometry has a conformal symmetry inherited from the isometries of the anti-de Sitter background. Upon fixing the world-volume diffeomorphism invariance these theories yield interacting conformal invariant field theories in 3,4 and 6 dimensions, respectively. There is a single coupling constant proportional to the $a d S$ radius of curvature $R$. Since $r$ is dimensionful, worldvolume vacua with $r \neq 0$ spontaneously break conformal invariance and are therefore degenerate. Each vacuum solution corresponds to an infinite planar brane at a distance $\langle r\rangle$ from the horizon, where $r$ is the Nambu-Goldstone field of spontaneously broken conformal invariance . The 'end of the universe' limit $\langle r\rangle \rightarrow \infty$ is also the free-field limit so our results encompass previous free-field constructions 4 , 5] as well as previous partial constructions in which only the field $r$ was considered [7, 8].

It seems likely that the methods used in this paper can be extended to the full superconformal theory. It may also be interesting to examine the $(d, 2)$ theories in greater detail. Our discussion has been entirely at the level of classical field theory. Whether the conformal invariance of brane actions exhibited here survives quantization is an interesting open problem.

\footnotetext{
${ }^{6}$ On a surface of constant time, in our coordinates, the proper distance to the horizon from any finite value of $r$ is infinite, but $r=0$ is reached for finite affine parameter on, say, a timelike geodesic. Thus $r$ can be considered as a measure of distance from the horizon.
} 


\section{Acknowledgments.}

We had stimulating discussions with E. Bergshoeff, H.J. Boonstra, M. Derix, M. Dine, M. Duff, S. Ferrara, E. Halyo, J. Maldacena, A. Rajaraman and E. Silverstein. The work of R. K. and J. K. is supported by the NSF grant PHY-9219345. The work of J. K. is also supported by the United States Department of Defense, NDSEG Fellowship program. Work is supported by the European Commission TMR programme ERBFMRX-CT96-0045. We thank the theory division of CERN for the hospitality and especially the organizers of the workshop "Non-Perturbative Aspects of Strings, Branes and Fields", during which the first part of this work was performed.

\section{A The brane at the end of the universe}

One result of this paper is a one parameter class of static solutions of the branewave equations in the adS background provided by a supergravity compactification. The parameter is a radial coordinate specifying the location of the brane. Static solutions of this kind were also sought in [2] but it was concluded there (for $p=2$ ) that such solutions exist only when the brane is located at spatial infinity ${ }^{7}$. The purpose of this appendix is to explain this apparent contradiction. Essentially, it is due to the fact that, because adS space admits more than one timelike Killing vector field, there is more than one possible meaning to the word 'static'. Each timelike Killing vector field corresponds to a class of coordinate systems in which static solutions are time-independent. Consequently, to understand why we find static solutions that were not found in [2] requires an understanding of the differences between the (horospherical) coordinates $\left(x^{m}, \phi\right)$ used here and the coordinates used in [2]. As before, we consider $a d S_{p+2}$ as the hypersurface $T_{1}=0$ $2.11)$ in $\mathbb{E}^{(p+1,2)}$ where the latter has cartesian coordinates $\left(X^{0}, X^{ \pm}, X^{k}\right)$ with $k=1, \ldots, p$.

Let for convenience

$$
X^{0}=T
$$

\footnotetext{
${ }^{7}$ Non-supersymmetric, and non-static, solutions describing 'finite radius' membranes were subsequently found in [27] but we believe that these are different from the $p=2$ solutions described here. In particular, we expect our solutions to be supersymmetric in the context of super $p$-brane actions. This point will be addressed elsewhere.
} 


$$
\begin{aligned}
X^{ \pm} & =S \pm X^{p+1} \\
r^{2} & =\sum_{k=1}^{p}\left(X^{k}\right)^{2}+\left(X^{p+1}\right)^{2} .
\end{aligned}
$$

The constraint $T_{1}=0$ now becomes

$$
T_{1}=-T^{2}-S^{2}+r^{2}+(w R)^{2}=0 .
$$

Whereas we solved the constraint in the main text by (2.13), we now consider (with $a=\frac{1}{w R}$ )

$$
\begin{aligned}
& T=a^{-1} \sin (a \tau)\left(1+a^{2} r^{2}\right)^{\frac{1}{2}}, \\
& S=a^{-1} \cos (a \tau)\left(1+a^{2} r^{2}\right)^{\frac{1}{2}},
\end{aligned}
$$

In these coordinates the metric is

$$
\begin{aligned}
d s^{2} & =-d T^{2}-d S^{2}+d r^{2}+r^{2} d \Omega_{p}^{2} \\
& =-\left(1+a^{2} r^{2}\right) d \tau^{2}+\left(1+a^{2} r^{2}\right)^{-1} d r^{2}+r^{2} d \Omega_{p}^{2},
\end{aligned}
$$

where $d \Omega_{p}^{2}$ is the $S O(p+1)$-invariant metric on the $p$-sphere. This is for $p=2$ the $a d S_{4}$ metric of [2].

The Wess-Zumino term of the M2 brane is determined by the 4-form (2.17)

$$
\Omega_{4}=-\frac{3 a^{2}}{4 !} \varepsilon_{\hat{m}_{0} \ldots \hat{m}_{4}} X^{\hat{m}_{0}} d X^{\hat{m}_{1}} d X^{\hat{m}_{2}} d X^{\hat{m}_{3}} d X^{\hat{m}_{4}} .
$$

In the coordinates (A.3) with

$$
\begin{aligned}
& X_{1}=r \cos \theta, \\
& X_{2}=r \sin \theta \cos \phi, \\
& X_{3}=r \sin \theta \sin \phi,
\end{aligned}
$$

this becomes

$$
\Omega_{4}=-3 a d \tau d r d \theta d \phi\left(r^{2} \sin \theta\right)=d\left(a r^{3} \sin \theta d \tau d \theta d \phi\right),
$$

and we recover the $A_{012}$ of [2].

The Dirac action is given by (2.23). In the gauge where we identify the world volume coordinates with $\{\tau, \theta, \phi\}$, the field on the world-volume that 
remains is $r(\tau, \theta, \phi)$. We can find the world-volume potential by taking $r$ to be constant or all derivatives of $r$ vanishing. The result for the world-volume potential is

$$
V(r)=a r^{3}-r^{2} \sqrt{1+a^{2} r^{2}} .
$$

There is therefore a force proportional to

$$
-V^{\prime}(r)=3 a r^{2}-2 r\left(1+a^{2} r^{2}\right)^{\frac{1}{2}}+a^{2} r^{2}\left(1+a^{2} r^{2}\right)^{-\frac{1}{2}},
$$

Except for $r=0$, this vanishes only for $r=\infty$ and we therefore recover the result of [2] (see eq. (15) there). This yields the 'membrane at the end of the universe'. The coordinates (A.3) cover all of $a d S_{p+2}$ apart from $r=0$ which is (for $p>0$ ) the usual coordinate singularity at the origin. The coordinate ranges are (except for $p=0$, in which case $r$ is unrestricted)

$$
0 \leq \tau<2 \pi, \quad 0<r<\infty
$$

The time coordinate $\tau$ is identified with period $2 \pi$. The global structure is shown in the Carter-Penrose diagram of Figure 1 in which each point is a $p$-sphere: the shaded region in Fig. 11 is $a d S_{p+2}$. Its universal cover is obtained by dropping the identification under time translations, in which case the diagram continues ad infinitum in both temporal directions. A typical (constant but finite $r$ ) orbit of the timelike killing vector field $\partial / \partial \tau$ is shown. Note that it necessarily cuts the null hypersurfaces $\mathcal{H}^{ \pm}$, the significance of which will become clear below. The main result of [2] can be summarised by saying that the worldvolume field configurations describing a static membrane (of topology $S^{p}$ ) at constant $r$ solves the relevant branewave equations only in the limit $r \rightarrow \infty$. However, this does not mean that there cannot be solutions that are static in terms of some other time coordinate.

In the horospherical coordinates used in the main text (2.13), $d s^{2}$ is singular at $\phi=0$, so that $\phi$ must be restricted to either the 'exterior' spacetime with $\phi>0$ or to the isometric 'interior' spacetime $\phi<0$. However, the singularity at $\phi=0$ is just a coordinate singularity, as the previously introduced coordinates demonstrate. The hypersurface $\phi=0$ is the union of a degenerate future Killing horizon (the null hypersurface $\mathcal{H}^{+}$marked on Fig. 1) and a degenerate past Killing horizon $\left(\mathcal{H}^{-}\right)$of the timelike Killing vector field $\partial / \partial t$. The 'exterior' spacetime is shown in Fig. 2 with a typical (constant but finite and non-zero $\phi$ ) orbit of the timelike vector field $\partial / \partial t$. As shown in the main text, a world-volume field configuration describing a static membrane 


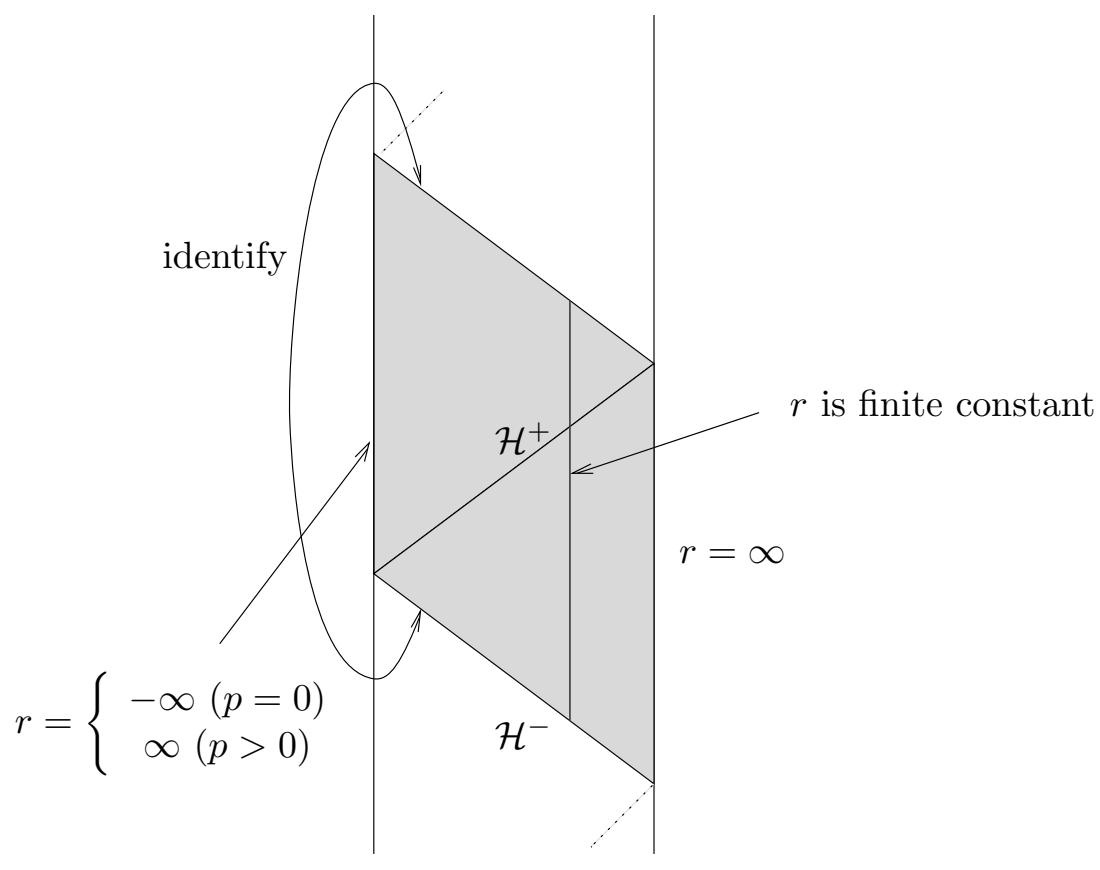

Figure 1: Carter Penrose diagram with global structure in parametisation $(\tau, r)$. Each point is a $p$-sphere. The shaded region is $a d S_{p+2}$.

at constant $\phi$ solves the relevant branewave equations for any (non-zero) $\phi$. This is consistent with the results of [2] because a constant $\phi$ hypersurface is static with respect to $\partial / \partial \tau$ only when the constant value of $\phi$ goes to infinity, and in this case the constant $\phi$ hypersurface coincides with the hypersurface $r=\infty$, except that the latter includes the points $i_{ \pm}$at infinity. Note that the range of $t$ is $(-\infty, \infty)$ and that the proper time from finite $t$ to $|t|=\infty$ on an orbit of $\partial / \partial t$ is infinite. A similar state of affairs holds for the $\sigma$ coordinates, and hypersurfaces of constant $\phi$ are globally isometric to $(p+1)$-dimensional Minkowski space. This remains true for the limiting case in which $\phi=\infty$, so the worldvolume of the 'membrane at the end of the universe' is actually $(p+1)$-dimensional Minkowski space. If the points at infinity implicit in the $r=\infty$ description of this hypersurface are included then the worldvolume is actually conformally compactified $(p+1)$-dimensional Minkowski space, which is topologically $S^{1} \times S^{p}$. We should point out (following [5], to which we refer for further references) that the mass terms required for conformal 


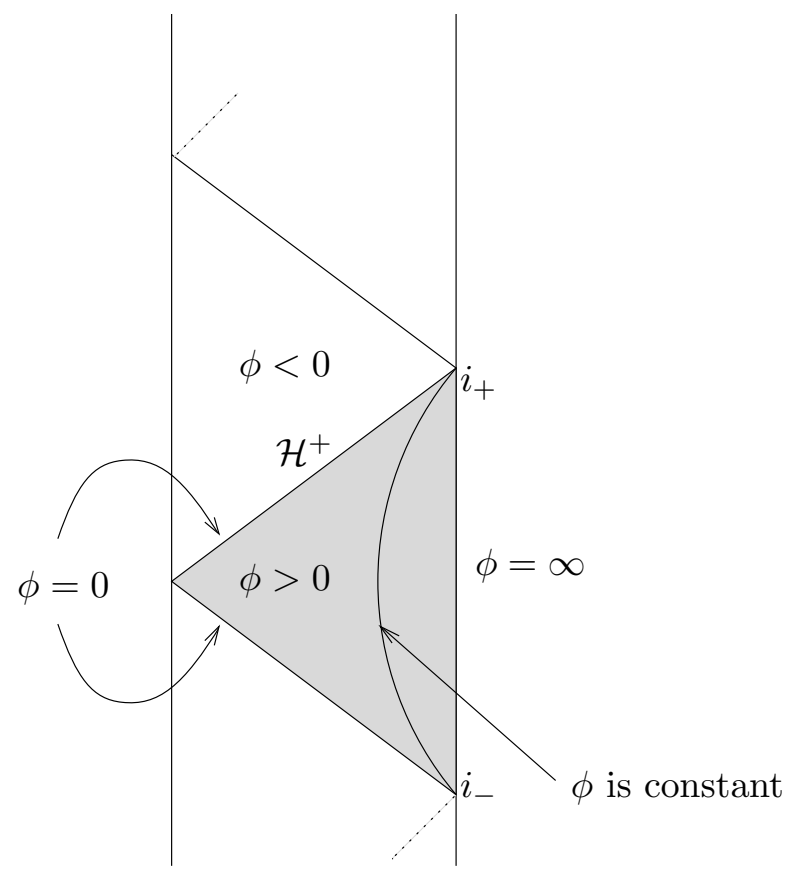

Figure 2: Carter Penrose diagram with global structure in horosperical coordinates. The shaded region is covered by horospherical coordinates for $\phi>0$.

field theories on $S^{1} \times S^{p}$ for finite radius p-sphere are absent in the infinite radius limit, consistent with the absence of such terms in the (interacting) conformal field theories found here.

\section{B Near-horizon supersymmetry of D1+D5}

Here we show that near the horizon the D1+D5 solution used in the main text preserves 16 supersymmetries, which is double the number admitted by the full D1+D5 solution of IIB supergravity. In the near-horizon geometry the dilaton is a constant, which we may set to zero. Since the axion (pseudoscalar) and all fermions also vanish, the IIB supersymmetry trans- 
formations reduce to

$$
\begin{aligned}
\delta \lambda & =\frac{3-p}{4(p+2) !} F_{\mu_{1} \ldots \mu_{p+2}} \gamma^{\mu_{1} \ldots \mu_{p+2}} \imath \epsilon^{*} \\
\delta \psi_{\mu} & =\partial_{\mu} \epsilon-\frac{1}{4} \omega_{\mu}^{\bar{a} \bar{b}} \gamma_{\bar{a}} \gamma_{\bar{b}} \epsilon+\frac{(-1)^{p}}{8(p+2) !} F_{\mu_{1} \ldots \mu_{p+2}} \gamma^{\mu_{1} \ldots \mu_{p+2}} \gamma_{\mu} \imath \epsilon^{*}
\end{aligned}
$$

Filling in the near-horizon solution given in $(3.28)$ it follows that

$$
\delta \lambda=\frac{\imath \gamma^{\bar{i}} x_{i}}{R r} \gamma^{0} \gamma^{5}\left(1-\gamma^{1234}\right) \epsilon^{*}
$$

where tangent-space indices are understood for the $\gamma$-matrices. Preservation of supersymmetry requires $\delta \lambda=0$. We see that this is satisfied by the 16 eigenspinors of $\gamma^{1234}$ with eigenvalue 1, i.e.

$$
\left(1-\gamma^{1234}\right) \epsilon_{k i l}^{*}=0
$$

To show that the D1+D5 solution admits 16 supersymmetries near its horizon we must now show that spinors satisfying (B.4) also solve $\delta \psi_{a}=0$. In the limit $r \rightarrow 0$ and using (B.4), it is straightforward to find

$$
\begin{aligned}
\delta \psi_{v} & \approx \partial_{v} \epsilon \\
\delta \psi_{r} & \approx \partial_{r} \epsilon \\
\delta \psi_{i} & \approx \partial_{i} \epsilon-\frac{1}{2} \frac{x_{i}}{r^{2}} \epsilon+\frac{1}{2} \gamma^{\bar{j}} \gamma_{i} \frac{x_{j}}{r^{2}} \epsilon-\frac{i}{2} \gamma^{\bar{j}} \gamma_{\bar{i}} \frac{x_{j}}{r^{2}} \gamma^{0} \gamma^{5} \epsilon^{*}
\end{aligned}
$$

where

$$
v \in[0,5] \quad r \in[1,2,3,4] \quad i, j \in[6,7,8,9] .
$$

Preservation of supersymmetry requires the variations of the gravitino to vanish and we find an explicit solution for the killing spinors

$$
\epsilon_{k i l}=\sqrt{\frac{r}{R}}\left(\epsilon_{0}+i \gamma^{0} \gamma^{5} \epsilon_{0}^{*}\right)
$$

where

$$
\left(1-\gamma^{1234}\right) \epsilon_{0}=0
$$

and $\epsilon_{0}$ constant. Therefore near the horizon the D1+D5 configuration preserves $1 / 2$ of supersymmetry.

\footnotetext{
${ }^{8}$ In this section we use the mostly minus signature and the conventions given in 28 .
} 


\section{Small velocity expansion}

In this appendix we give the derivation of the expansion of the world-volume actions for M2, D3 and M5, in the limit of small velocities. We introduce flat indices $\bar{\mu}$, raised and lowered with $\eta_{\bar{\mu} \bar{\nu}}$. To convert curved indices to flat ones we use a ' $\delta$-function vielbein', i.e.

$$
T_{\mu \nu}=\delta_{\mu}^{\bar{\mu}} \delta_{\nu}^{\bar{\nu}} T_{\bar{\mu} \bar{\nu}} .
$$

In the sequel these $\delta$-functions will be omitted. The main difficulty is the expansion of

$$
\operatorname{det}\left(\delta^{\mu}{ }_{\nu}+X^{\mu}{ }_{\nu}\right)
$$

where $X_{\nu}^{\mu}$ is a matrix depending on the case considered. The general form of the 'Dirac' part, or generalizations thereof, of the brane actions that we have considered is

$$
I=I_{D}+I^{\prime} ; \quad I_{D}=-\int d^{p+1} \sigma\left(\frac{r}{R}\right)^{\frac{p+1}{w}} \sqrt{\operatorname{det}(1+X)},
$$

with

$$
\begin{aligned}
X_{\bar{\nu}}^{\bar{\mu}} & =A_{\bar{\nu}}^{\bar{\mu}}+B_{\bar{\nu}}^{\bar{\mu}}, \\
A_{\bar{\nu}}^{\bar{\mu}} & =\left(\frac{R}{r}\right)^{\frac{p+1}{w}} \partial^{\bar{\mu}} X^{m^{\prime}} \partial_{\bar{\nu}} X_{m^{\prime}},
\end{aligned}
$$

where $B_{\bar{\mu} \bar{\nu}}$ is proportional to $F_{\mu \nu}$ or $\mathcal{H}_{\mu \nu}^{*}$, hence antisymmetric. To go to the limit of small velocities we note that $A$ is of order two in velocities and $B$ of order one. $I^{\prime}$ is only non zero for M5, see below. Up to order four in velocities we have

$$
\begin{aligned}
\operatorname{tr} X & \approx \operatorname{tr} A, \\
\operatorname{tr} X^{2} & \approx \operatorname{tr} A^{2}+\operatorname{tr} B^{2}, \\
\operatorname{tr} X^{3} & \approx 3 \operatorname{tr}(A B B), \\
\operatorname{tr} X^{4} & \approx \operatorname{tr} B^{4} .
\end{aligned}
$$

Using the series-expansion for $\sqrt{\operatorname{det}(1+X)}$ we obtain,

$$
\begin{aligned}
\sqrt{\operatorname{det}(1+X)}= & 1+\frac{1}{2} \operatorname{tr} A^{2}-\frac{1}{4} \operatorname{tr} B^{2} \\
& -\frac{1}{4}\left[\operatorname{tr} A^{2}-\frac{1}{2}(\operatorname{tr} A)^{2}\right]-\frac{1}{8}\left[\operatorname{tr} B^{2}-\frac{1}{4}\left(\operatorname{tr} B^{2}\right)^{2}\right] \\
& +\frac{1}{2}\left[\operatorname{tr}(A B B)-\frac{1}{4} \operatorname{tr} A \operatorname{tr} B^{2}\right],
\end{aligned}
$$


up to fourth order in velocities. So in all cases we find that

$$
\begin{aligned}
I_{D}=-\int d^{p+1} x\left(\frac{r}{R}\right)^{\frac{p+1}{w}} & \left(1+\frac{1}{2} \operatorname{tr} A-\frac{1}{4} \operatorname{tr} B^{2}\right. \\
& -\frac{1}{4}\left[\operatorname{tr} A^{2}-\frac{1}{2}(\operatorname{tr} A)^{2}\right] \\
& -\frac{1}{8}\left[\operatorname{tr} B^{4}-\frac{1}{4}\left(\operatorname{tr} B^{2}\right)^{2}\right] \\
& \left.+\frac{1}{2}\left[\operatorname{tr}(A B B)-\frac{1}{4} \operatorname{tr} A \operatorname{tr} B^{2}\right]+\ldots\right) .
\end{aligned}
$$

For M2 and D3 we have

$$
\begin{aligned}
\text { M2 } \quad A_{\bar{\nu}}^{\bar{\mu}}=\left(\frac{R}{r}\right)^{6} \partial^{\bar{\mu}} X^{m^{\prime}} \partial_{\bar{\nu}} X_{m^{\prime}} \quad B_{\bar{\nu}}^{\bar{\mu}}=0 \\
\text { D3 } \quad A_{\bar{\nu}}^{\bar{\mu}}=\left(\frac{R}{r}\right)^{4} \partial^{\bar{\mu}} X^{m^{\prime}} \partial_{\bar{\nu}} X_{m^{\prime}} \quad B_{\bar{\nu}}^{\bar{\mu}}=\left(\frac{R}{r}\right)^{2} \eta^{\bar{\mu} \bar{\rho}} F_{\bar{\rho} \bar{\nu}} .
\end{aligned}
$$

This yields the formulae of the main text. The M5 brane has to be treated separately, due to the many hidden metrics in the definitions of the fields that appear in the action. But in the end everything works out nicely. We take $w=2$. The inverse metric

$$
\begin{aligned}
g^{\mu \nu} & =\left(\frac{R}{r}\right)\left(\delta_{\bar{\rho}}^{\bar{\mu}}+\sum_{n=1}^{\infty}(-)^{n}\left(A^{n}\right)_{\bar{\rho}}^{\bar{\mu}}\right) \eta^{\bar{\rho} \bar{\nu}} \\
& \approx\left(\frac{R}{r}\right)\left(\eta^{\bar{\mu} \bar{\nu}}-A^{\bar{\mu} \bar{\nu}}+\ldots\right)
\end{aligned}
$$

up to second order in velocities (note that since this metric is used to contract indices of fields we only need it to this order). $A_{\bar{\nu}}^{\bar{\mu}}$ is given in (C.3). Taking the definitions in (5.3) of [6] and expanding the metrics, we have

$$
\begin{aligned}
\sqrt{g} & \approx 1+\frac{1}{2} A^{\bar{\mu}} \\
u^{2} & =u_{\mu} g^{\mu \nu} u_{\nu} \approx\left(\frac{R}{r}\right)\left(u_{\bar{\mu}} \eta^{\bar{\mu} \bar{\nu}} u_{\bar{\nu}}-u_{\bar{\mu}} A^{\bar{\mu} \bar{\nu}} u_{\bar{\nu}}\right), \\
u_{\bar{\mu}} & =\bar{\mu}_{\bar{\mu}} a \\
v_{\rho} & \equiv \frac{u_{\rho}}{\sqrt{u^{2}}} \approx\left(\frac{r}{R}\right)^{1 / 2} v_{\bar{\rho}}\left(1+\frac{1}{2} v_{\bar{\mu}} A^{\bar{\mu} \bar{\nu}} v_{\bar{\nu}}\right), \\
\mathcal{H}^{* \mu \nu} & =\frac{1}{6 \sqrt{g}} \varepsilon^{\mu \nu \rho \sigma \tau \phi} v_{\rho} \mathcal{H}_{\sigma \tau \phi} \approx\left(\frac{R}{r}\right)^{5 / 2} \mathcal{H}^{* \mu \bar{\nu}}\left(1+\frac{1}{2} v_{\bar{\rho}} A^{\bar{\rho} \bar{\sigma}} v_{\bar{\sigma}}-\frac{1}{2} A^{\bar{\rho}}\right), \\
\mathcal{H}_{\mu \nu}^{*} & \approx\left(\frac{R}{r}\right)^{1 / 2}\left(\mathcal{H}^{* \bar{\mu} \bar{\nu}}\left(1+\frac{1}{2} v_{\bar{\rho}} A^{\bar{\rho} \bar{\sigma}} v_{\bar{\sigma}}-\frac{1}{2} A_{\bar{\rho}}^{\bar{\rho}}\right)+A_{\bar{\mu} \bar{\rho}} \mathcal{H}^{* \bar{\rho}} \overline{\bar{\nu}}+\mathcal{H}_{\bar{\mu} \bar{\rho}}^{*} A_{\bar{\nu}}^{\bar{\rho}}\right),
\end{aligned}
$$




$$
\mathcal{H}_{\mu \nu} \equiv g^{\rho \sigma} v_{\rho} \mathcal{H}_{\sigma \mu \nu} \approx\left(\frac{R}{r}\right)^{1 / 2}\left(\mathcal{H}_{\bar{\mu} \bar{\nu}}-v_{\bar{\rho}} A^{\bar{\rho} \bar{\sigma}} \mathcal{H}_{\bar{\sigma} \bar{\mu} \bar{\nu}}+\frac{1}{2} v_{\bar{\rho}} A^{\bar{\rho} \bar{\sigma}} v_{\bar{\sigma}} \mathcal{H}_{\bar{\mu} \bar{\nu}}\right)
$$

On the right hand side of the $\approx$-sign every index is contracted with $\eta_{\bar{\mu} \bar{\nu}}$. For $I^{\prime}$, which is the last term of (3.20), this gives

$$
\begin{aligned}
I^{\prime}=-\frac{1}{4} \int d^{6} \sigma \sqrt{-\operatorname{det} g} \mathcal{H}^{* \mu \nu} \mathcal{H}_{\mu \nu} & \\
\approx-\frac{1}{4} \int d^{6} \sigma \mathcal{H}^{* \mu \bar{\mu}} \mathcal{H}_{\bar{\mu} \bar{\nu}} & \\
& \quad-\frac{1}{4} \mathcal{H}^{* \bar{\mu} \bar{\nu}}\left(v_{\bar{\rho}} A^{\bar{\rho} \bar{\sigma}} v_{\bar{\sigma}} \mathcal{H}_{\bar{\mu} \bar{\nu}}-v_{\bar{\rho}} A^{\bar{\rho} \bar{\sigma}} \mathcal{H}_{\bar{\sigma} \bar{\mu} \bar{\nu}}\right) .
\end{aligned}
$$

Now we can combine everything and use formula (C.6) with

$$
\begin{aligned}
& A_{\bar{\nu}}^{\bar{\mu}}=\left(\frac{R}{r}\right)^{3} \partial^{\bar{\mu}} X^{m^{\prime}} \partial_{\bar{\nu}} X_{m^{\prime}}, \\
& B_{\bar{\nu}}^{\bar{\mu}}=i\left(\frac{R}{r}\right)^{3 / 2}\left[\mathcal{H}_{\bar{\nu}}^{* \bar{\nu}}\left(1+\frac{1}{2} v_{\bar{\rho}} A^{\bar{\rho} \bar{\sigma}} v_{\bar{\sigma}}-\frac{1}{2} A_{\bar{\rho}}^{\bar{\rho}}\right)+A^{\bar{\mu} \bar{\rho}} \mathcal{H}_{\bar{\rho} \bar{\nu}}^{*}+\mathcal{H}^{* \bar{\mu} \bar{\rho}} A_{\bar{\rho} \bar{\nu}}\right],
\end{aligned}
$$

together with (C.10) to obtain the formula of the main text.

\section{References}

[1] C. Fronsdal, Singletons, in proceedings of the 1989 Latin American School on Strings and Fundamentals, preprint UCLA/89/TEP/66.

[2] E. Bergshoeff, M.J. Duff, C.N. Pope and E. Sezgin, Supersymmetric supermembrane vacua and singletons, Phys. Lett. 199B (1987) 69; Compactifications of the eleven-dimensional supermembrane, Phys. Lett. 224B (1989) 71.

[3] G. W. Gibbons and P. K. Townsend, Vacuum interpolation in supergravity via super p-branes, Phys. Rev. Lett. 71 (1993) 3754 ; hep-th/9307049.

[4] H. Nicolai and E. Sezgin, Singleton representations of $O S p(N \mid 4)$, Phys. Lett. 143B (1984) 389; 
M. Günaydin, B.E.W. Nilsson, G. Sierra and P.K. Townsend, Singletons and superstrings, Phys. Lett. 176B (1986) 45;

E. Bergshoeff, A. Salam, E. Sezgin and Y. Tanii, $N=8$ supersingleton quantum field theory, Nucl. Phys. B305 (1988) 497;

H. Nicolai, E. Sezgin and Y. Tanii, Conformally invariant supersymmetric field theories on $S^{p} \times S^{1}$ and super $p$-branes, Nucl. Phys. B305 (1988) 483.

[5] M.P. Blencowe and M.J. Duff, Supersingletons, Phys. Lett. 203B (1988) 229.

[6] P. Claus, R. Kallosh and A. Van Proeyen, M 5-brane and superconformal $(0,2)$ tensor multiplet in 6 dimensions, Nucl. Phys. $\mathbf{B}$, to be published, hep-th/9711161.

[7] J. Maldacena, The large $N$ limit of superconformal field theories and supergravity, hep-th/9711200.

[8] R. Kallosh, J. Kumar and A. Rajaraman, Special conformal symmetry of worldvolume actions, hep-th/9712073.

[9] S. Ferrara and C. Fronsdal, Conformal Maxwell theory as a singleton field theory on ad $S_{5}$, IIB three branes and duality, hep-th/9712239.

[10] M.J. Duff, G.W. Gibbons and P.K. Townsend, Macroscopic superstrings as interpolating solitons, Phys. Lett. 332 B (1994) 321;

G.W. Gibbons, G.T. Horowitz and P.K. Townsend, Higher-dimensional resolution of dilatonic black hole singularities, Class. Quantum Grav. 12 (1995) 297.

[11] C. Vafa, Evidence for F-theory, Nucl. Phys. B469 (1996) 403, hepth/9602022;

C.M. Hull, String dynamics at strong coupling, Nucl. Phys. B468 (1996) 113, hep-th/9512181;

S. Hewson, An approach to F-theory, hep-th/9712017.

[12] I. Bars, S-theory, Phys. Rev. D55 (1997) 2373, hep-th/9607112.

[13] I. Rudychev, E. Sezgin and P. Sundell, Supersymmetry in dimensions beyond eleven, hep-th/9711127. 
[14] M. Blencowe and M.J. Duff, Supermembranes and signature of spacetime, Nucl. Phys. B310 (1988) 387;

D. Kutasov and E. Martinec, M-branes and N=2 strings, Class. Quantum Grav. 14 (1997) 2483;

S. Hewson and M. Perry, The Twelve dimensional super (2+2)-brane, Nucl.Phys. B492 (1997), 249, hep-th/9612008.

[15] S. Hyun, U-duality between Three and Higher Dimensional Black Holes, hep-th/9704005.

[16] R. Kallosh and J. Kumar, Supersymmetry enhancement of D-p-branes and M-branes, Phys. Rev. D56 (1997) 4934, hep-th/9704189.

[17] H.J. Boonstra, B. Peeters and K. Skenderis, Duality and asymptotic geometries, Phys. Lett. 411B (1997) 59, hep-th/9706192; Branes and anti-de Sitter space-times, to appear in the proceedings of the conference "Quantum Aspects of Gauge theories, Supersymmetry and Unification", Neuchatel, September 1997, hep-th/9801076; K. Sfetsos and K. Skenderis, Microscopic derivation of the Bekenstein-Hawking entropy formula for non-extremal black holes, Nucl. Phys. B to be published, hep-th/9711138.

[18] P.M. Cowdall and P.K. Townsend, Gauged supergravity vacua from intersecting branes, hep-th/9801165.

[19] W. Nahm, Supersymmetries and their representations, Nucl. Phys. B135 (1978) 149.

[20] M. Günaydin, G. Sierra and P.K. Townsend, The unitary supermultiplets of $d=3$ anti-de Sitter and $d=2$ conformal superalgebras, Nucl. Phys. B274 (1986) 429;

M. Günaydin, B.E.W. Nilsson, G. Sierra and P.K. Townsend, Singletons and superstrings, Phys. Lett. 176B (1986) 45.

[21] E. Cremmer, I.V. Lavrinenko, H. Lu, C.N. Pope, K.S. Stelle and T.A. Tran, Euclidean signature supergravities, dualities and instantons, hepth/9803259.

[22] P. Claus, M. Derix, R. Kallosh, J. Kumar, P.K. Townsend and A. Van Proeyen, Black holes and superconformal mechanics, hep-th/9804177. 
[23] I. Bandos, K. Lechner, A. Nurmagambetov, P. Pasti, D. Sorokin and M. Tonin, Covariant action for the super-five-brane of M-theory, Phys. Rev. Lett. 78 (1997) 4332.

[24] C.G. Callan and J.M. Maldacena, D-brane approach to black hole quantum mechanics, Nucl. Phys. B472 (1996) 591, hep-th/9602043.

[25] A. Tseytlin, Extreme dyonic black holes in string theory, Mod. Phys. Lett. A11 (1996) 689, hep-th/9601177.

[26] Fr. Brandt, J. Gomis and J. Simón, The rigid symmetries of bosonic D-strings, hep-th/9803196.

[27] E. Bergshoeff, M.J. Duff, C.N. Pope and E. Sezgin, Compactifications of the eleven-dimensional supermembrane, Phys. Lett. 224B (1989) 71.

[28] E. Bergshoeff, P-Branes, D-Branes and M-Branes, in 'Gauge Theory Applied Supersymmetry and Quantum Gravity II', eds. A. Sevrin, K.S. Stelle, K. Thielemans and A. Van Proeyen, Imperial College Press, p. 210, hep-th/9611099. 